\title{
Innovations in Eye Care: Sport Vision
}

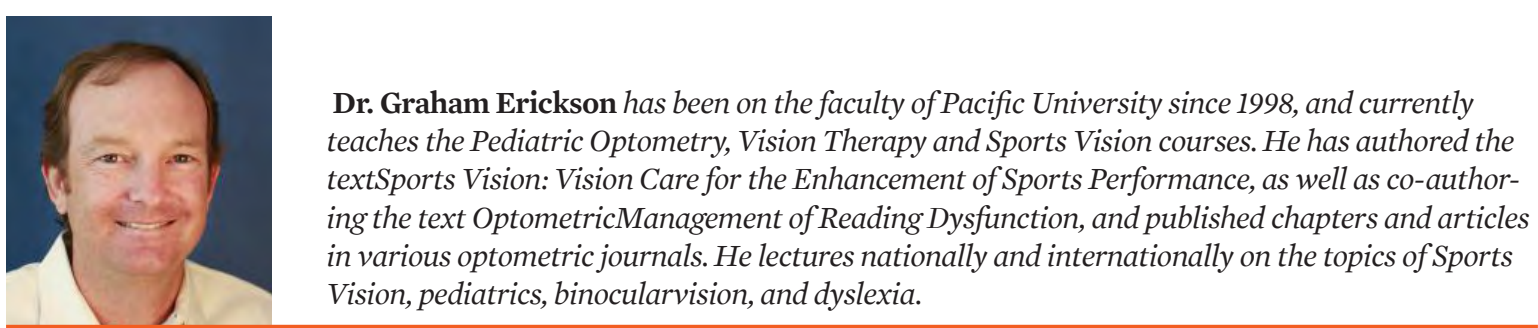

$\mathrm{O}$ ptometry has a long tradition of providing vision care services to optimize daily function. While this has often focused on academic and occupational performance, sports performance is another important area where vision plays a critical role. Athletes, trainers and coaches have recognized that excellent vision is an important aspect of performance, and more athletes and teams are looking for ways to optimize visual performance. While an interest in sports vision is not new, there have been some recent innovations in instrumentation that utilize new digital technology.

VISUAL PERFORMANCE ASSESSMENT

Many studies have found that higher-achieving athletes perform better on many measures of visual performance than non-athletes or lower-achieving athletes. Recently, several companies have developed instruments that measure various aspects of visual performance, and such performance can be compared to a database of performance by other athletes. Computerized assessment and training devices such as the Senaptec Sensory Station (http:// senaptec.com), Sports Vision Performance from M\&S ${ }^{\circledR}$ (http://www.mstech-eyes.com/products/category/sportsvision-performance), RightEye (https://www.righteye.com/tests-therapies/vision-performance) and Vizual Edge Performance Trainer $^{\circledR}$ (http://vizualedge.com) have been developed to measure a broad range of visual, cognitive and sensorimotor skills.

The Senaptec Sensory Station is a successor to the Sensory Station device originally developed by Nike Inc. Research with the Nike version of this instrument has demonstrated that certain assessments in the battery are reliable and cross-validated measures that can be used to investigate sensorimotor abilities in relation to performance in sports. ${ }^{1,2}$ Furthermore, worse performance on the Sensory Station has been associated with an increased likelihood of sustaining head impacts during practices and games among US collegiate football players, ${ }^{3}$ indicating a link between collision avoidance and visual-motor skills. This suggests that these assessments might be useful for proactively assessing the risk of concussion, as well as potentially measuring the recovery of visual performance following a concussive episode. While there is limited evidence regarding the reliability or validity of the other systems, many of these systems employ standard psychophysical protocols, and thus it is reasonable to expect that the measurements are reliable.

\section{SPORTS VISION TRAINING}

Sports vision training (SVT) programs operate under the logic that practice with demanding visual, perceptual and sensorimotor tasks will improve vision, leading to quicker sensory processing, swifter and more accurate motor movements, and improved athletic performance while also potentially reducing injury. SVT approaches have been advanced greatly by training programs that use information about the structure and function of the visual system combined with recent innovations in perceptual learning paradigms to engender more specific and robust learning. Virtual reality (VR) simulations that can recreate and augment sporting contexts to promote certain sports-specific visual-cognitive abilities have also enhanced SVT approaches. 
A recent innovation in visual component training is called Ultimeyes ${ }^{\circledR}$ (https://ultimeyesvision.com). This video application incorporates diverse stimuli, adaptive near-threshold training with learning-optimized flickering stimuli, and multisensory feedback in a digital training program designed to improve foundational aspects of visual sensitivity. In a series of studies, this training app has been shown to improve visual acuity and contrast sensitivity in both non-athletes ${ }^{4}$ and athletes, ${ }^{5}$ as well as to improve batting ${ }^{5}$ and pitching ${ }^{6}$ performance in collegiate baseball players.

The CogniSense NeuroTracker (https://neurotracker.net) is an example of a perceptual-cognitive training program. The training platform entails an immersive three-dimensional "multiple object tracking" program to increase cognitive load. There has been ample research with the NeuroTracker system in groups of healthy young adults, ${ }^{7}$ healthy older adults, ${ }^{8,9}$ and athletes across several sports and skill levels. NeuroTracker performance has been correlated with actual game performance in professional basketball players, ${ }^{10}$ and training with this program has been demonstrated to selectively transfer to improved small-sided game performance in university-level soccer players. ${ }^{11}$

Actual sports practice is typically viewed as the most natural method for developing the necessary skills for success. However, practice sessions have the potential for injury to the athlete. Over the past several years, computerized simulations and VR platforms have been developed to simulate game action, and are now considered a type of natural sports training. Such simulation platforms allow for the design of complex training protocols that can mimic real-game activities, allowing athletes to gain 'mental repetitions.' Three companies in particular, Eon Sports VR (http://eonsportsvr.com/), StriVR Labs (http://www.strivrlabs.com), and Axon Sports (http://www.axonsports. com/), have recently developed suites of digital training simulations that are marketed towards athletes, coaches and trainers. In addition to these broad commercial platforms that have applications for many different sports, there is a growing number of products that target specific individual sports. It is important to note that these VR sport simulations are a new technology with relatively little supporting evidence at this time.

\section{SUMMARY}

This brief summary has highlighted some of the recent innovations in sports vision. More detailed information can be found in a recently published review paper. ${ }^{12}$ These innovations provide options to help your athletic patients see their sport more clearly.

\section{References}

1. Erickson GB, Citek K, Cove M, et al. Reliability of a computer-based 7 . system for measuring visual performance skills. Optometry 2011, 82(9):528-42.

2. Wang L, Krasich K, Bel-Bahar T, Hughes L, Mitroff SR, Appelbaum LG. Mapping the structure of perceptual and visual-motor abilities in healthy young adults. Acta Psychol (Amst) 2015; 157:74-84.

3. Harpham JA, Mihalik JP, Littleton AC, Frank BS, Guskiewicz KM. The effect of visual and sensory performance on head impact biomechanics in college football players. Ann Biomed Eng 2014, 42:1-10.

4. Deveau J, Lovcik G, Seitz AR. Broad-based visual benefits from training with an integrated perceptual-learning video game. Vision Res 2014; 99:134-40.

5. Deveau J, Ozer DJ, Seitz AR. Improved vision and on-field performance in baseball through perceptual learning. Curr Biol 2014; 24(4):R146-7.

6. Deveau J, Thurman S, Seitz AR. Improvements in baseball pitching through vision training. In press
Parsons B, Magill T, Boucher A, et al. Enhancing cognitive function using perceptual-cognitive training. Clinical EEG and Neuroscience 2016; 47:37-47.

8. Legault I, Allard R, Faubert J. Healthy older observers show equivalent perceptual-cognitive training benefits to young adults for multiple object tracking. Front Psychol 2013; 4:323.

9. Legault I, Faubert J. Perceptual-cognitive training improves biological motion perception: Evidence for transferability of training in healthy aging. Neuroreport 2012; 23:469-73.

10. Mangine GT, Hoffman JR, Wells AJ, et al. Visual tracking speed is related to basketball-specific measures of performance in NBA players. J Strength Conditioning Res 2014; 28:2406-14.

11. Romeas T, Faubert J. Soccer athletes are superior to non-athletes at perceiving soccer specific and non-sport specific human biological motion. Front Psychol 2015; 6:705.

12. Appelbaum LG, Erickson G. Sports vision training: A review of the state-of-the-art in digital training techniques. Int Rev Sport Exercise Psychol 2016; DOI: 10.1080/1750984X.2016.1266376 